\title{
A near projective plane of order 6
}

page 1 / 9

go back

full screen

close

quit

\author{
Alan R. Prince
}

Abstract

We construct a near projective plane of order 6 containing 15 pure lines by extending the dual of the point-line geometry of $\mathrm{PG}(3,2)$.

Keywords : projective plane of order 6 , spreads of PG(3,2), Conwell heptads MSC 2000: 05B25, 51E30

\section{Introduction}

A near projective plane of order $n$ is a point-line incidence structure with $\left(n^{2}+\right.$ $n+1)$ points and lines, each line incident with $(n+1)$ points and each point incident with $(n+1)$ lines, in which any two distinct lines intersect in at most two points and any two distinct points are both incident with at most two lines. Any projective plane of order $n$ is clearly a near projective plane of order $n$. The dual of a near projective plane of order $n$ is also a near projective plane of order $n$. In a near projective plane, if the number of lines meeting a given line $\ell$ in $0,1,2$ points is $x, y, z$ respectively, then counting in two different ways the number of flags $(P, m)$, where $P$ is a point on $\ell$ and $m$ is a line through $P$ with $m \neq \ell$, gives $y+2 z=n(n+1)$. Since $x+y+z=n^{2}+n$, it follows that $x=z$. Thus, in a near projective plane, if the number of lines intersecting a given line in two points is $\lambda$, then the number of lines not intersecting the given line is also $\lambda$. A line with $\lambda=0$ is called a pure line. If all lines are pure, then we have a projective plane.

Near projective planes are relatively easy to construct from sets which are almost difference sets. For example, a near projective plane of order 6 can be constructed by expanding $\{0,1,2,6,14,17,24\}$ modulo 43 . However, such examples are cyclic and have no pure lines (unless they are projective planes). 

(b) the unordered pairs $i j$ from $\{1,2, \ldots, 7\}$;

(c) the unordered triples defining the lines of the Fano plane $F$.

Thus, we have a total of 43 lines: fifteen of type (a), twenty-one of type (b) and seven of type (c).

The points of the incidence structure are of three types:

(a) the unordered triples from $\{1,2, \ldots, 7\}$;

(b) the 3-cycles ordering the lines of $F$;

(c) the Fano plane $F$ itself.

Thus, we have a total of 43 points: thirty-five of type (a), seven of type (b) and a single point of type (c).

Incidence is defined as follows. A line of type (a) is incident precisely with the seven points of type (a), given by the seven triples of the corresponding Fano plane. A line $i j$ of type (b) is incident precisely with the five points of type (a) corresponding to the triples containing $i j$ and with two points of type (b) to be defined. A line $i j k$ of type (c) is incident precisely with the five points of type (a), given by the triple $i j k$ and the triples disjoint to $i j k$, with the point of type (b) given by the 3 -cycle ordering the line $i j k$ and with the unique point of type (c). Incidence between lines and points of type (b) is defined as follows. The cyclic ordering ( $p q r)$ of the points of the line $p q r$ of $F$ induces the following ordering on each pair of points of the line: $(p, q),(q, r),(r, p)$. Identifying the lines of type (b) with ordered pairs, the line $(i, j)$ of type (b) is incident with the point ( $p q r)$ of type (b) if and only if $i \in\{p, q, r\}$ and $j \notin\{p, q, r\}$. Thus, the line $(i, j)$ is incident with two points of type (b), given by the 3 -cycles ordering the further two lines through $i$, other than the line joining $i$ and $j$. The point (pqr) is incident with six lines of type (b), given by the ordered pairs with first component one of $p, q, r$ and second component not one of $p, q, r$.

As described in Section 2, the points of type (a) can be identified with the lines of $P G(3,2)$ and the lines of type (a) can be identified with the points of $P G(3,2)$. Thus, the lines and points of type (a), with the incidence defined, form an incidence structure isomorphic to the dual of the point-line geometry of $\operatorname{PG}(3,2)$. Furthermore, the lines of type (b) and (c) can be identified with spreads of $\operatorname{PG}(3,2)$ and the defined incidence of these lines with points of type (a) corresponds to the natural incidence of lines and spreads of $\mathrm{PG}(3,2)$ (a line is incident with a spread if the line is contained in the spread).

The following observation on the structure of the Fano plane $F$ is useful. If $i j k$ is a line of $F$ and $a, b, c, d$ are the points not on $i j k$, then $a b c, a b d, a c d, b c d$ are triangles of $F$ (sets of three points, no three collinear). Moreover, given any triangle $p q r$, there is a unique line not containing $p, q, r$. Thus, the 28 

[6] P. Erdös, C. Ko and R. Rado, Extremal problems among subsets of a set, Q. J. Math. 12 (1961), 313-318.

[7] M. Hall and R. Roth, On a conjecture of R.H. Bruck, J. Combin. Theory Ser. A 37 (1984), 22-31.

[8] J.W.P. Hirschfeld, Finite Projective Spaces of Three Dimensions, Oxford University Press, Oxford, 1985.

[9] A.R. Prince, Projective planes of order 12 and PG(3,3), Discrete Math. 208/209 (1999), 477-483.

[10] Covering sets of spreads in PG(3, q), Discrete Math. 238 (2001), 131-136.

Alan R. Prince

MaXwell Institute for Mathematical Sciences, Heriot-Watt University, Edinburgh EH14 4AS, SCOTLAND

e-mail: a.r.prince@hw.ac.uk 